\title{
Erratum to: The prevalence and clinical correlates of metabolic syndrome in patients with schizophrenia: findings from a cohort in Turkey
}

M. K. Yazıcı • A. E. Anıl Yağcıŏ̆lu •

A. Ertuğrul • N. Eni · S. Karahan • E. Karaağaoğlu •

S. L. Tokgözoğlu

Published online: 27 November 2010

(C) Springer-Verlag 2010

\section{Erratum to: Eur Arch Psychiatry Clin Neurosci DOI 10.1007/s00406-010-0118-x}

Unfortunately Figs. 1, 2, 3 and 4 were published with errors. The corrected figures are given here.

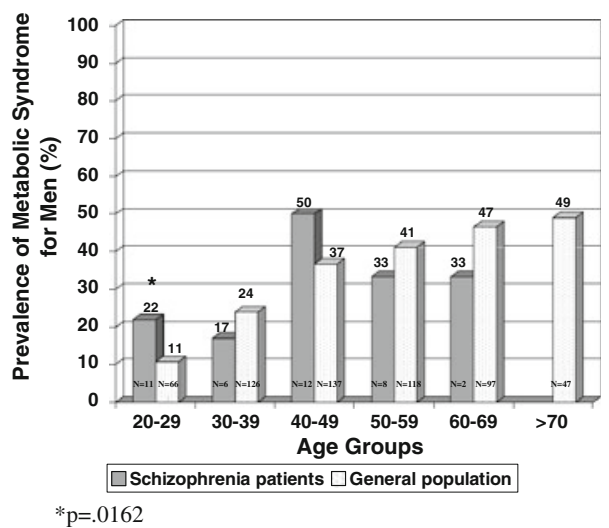

Fig. 1 Prevalence of metabolic syndrome for men in patients with schizophrenia and general population (METSAR study) [39] according to the ATP criteria. $* p=.0162$

The online version of the original article can be found under doi:10.1007/s00406-010-0118-x.

M. K. Yazıcı $(\bowtie)$ · A. E. Anıl Yağcioğlu · A. Ertuğrul · N. Eni

Department of Psychiatry, Hacettepe University

Faculty of Medicine, 06100 Ankara, Turkey

e-mail: kyazici@hacettepe.edu.tr

\section{S. Karahan · E. Karaağaoğlu}

Department of Biostatistics, Hacettepe University

Faculty of Medicine, 06100 Ankara, Turkey

S. L. Tokgözoğlu

Department of Cardiology, Hacettepe University

Faculty of Medicine, 06100 Ankara, Turkey
In addition, the reference number of Mc Evoy et al. in Table 1 was corrected to [16].

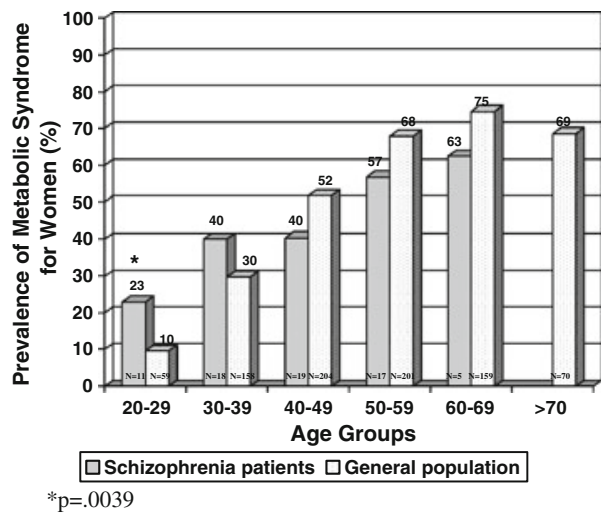

Fig. 2 Prevalence of metabolic syndrome for women in patients with schizophrenia and general population (METSAR study) [39] according to the ATP criteria. $* p=.0039$

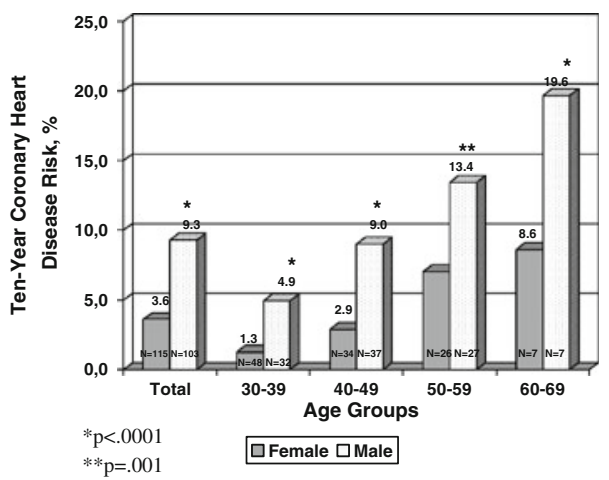

Fig. 3 Effect of sex on 10-year coronary heart disease risk in patients with schizophrenia. $* p<.0001 ; * * p=.001$ 


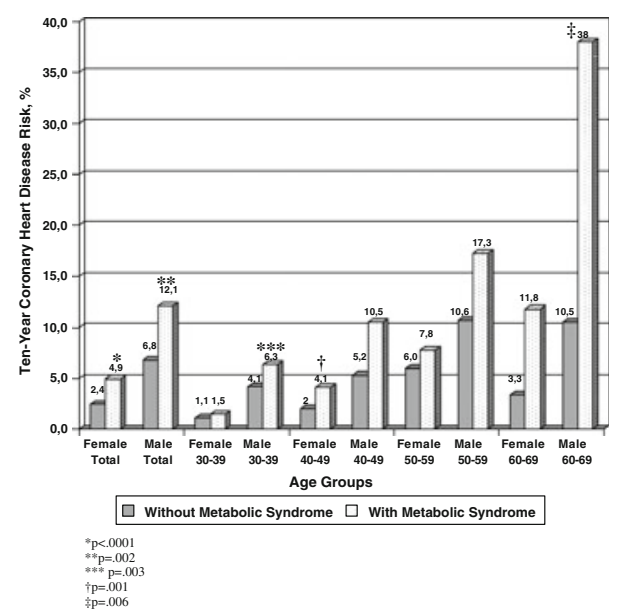

Fig. 4 Effect of metabolic syndrome by sex and age on 10-year coronary heart disease risk in schizophrenia. ${ }^{*} p<.0001 ; * * p=.002$; $* * * p=.003 ;{ }^{\dagger} p=.001 ;{ }^{*} p=.006$

Table 1 Prevalence estimates of the metabolic syndrome in patients with schizophrenia and schizoaffective disorder in different countries [8-36]

\begin{tabular}{|c|c|c|c|c|c|c|}
\hline Study & Country & Sample & $N$ (female/male) & Mean age (years) & Criteria & Prevalence \\
\hline Heiskanen et al. [8] & Finland & Outpatients & $35(16 / 19)$ & 44.5 & ATP-III & $37.1 \%$ \\
\hline Littrell et al. [9] & USA and Taiwan & In-outpatients & $\begin{array}{l}\text { USA } 98(37 / 61) \\
\text { Taiwan } 27(13 / 14)\end{array}$ & $\begin{array}{l}\text { USA } 41.8 \\
\text { Taiwan } 42\end{array}$ & ATP-III & $\begin{array}{l}\text { USA } 51 \% \\
\text { Taiwan } 22.2 \%\end{array}$ \\
\hline Kato et al. [10] & USA & Outpatients & $48(24 / 24)$ & 40.3 & ATP-III & $63.0 \%$ \\
\hline Basu et al. [11] & USA & Outpatients & $33(19 / 14)$ & 44.5 & ATP-III & $42.4 \%$ \\
\hline Cohn et al. [12] & Canada & In-outpatients & $240(84 / 156)$ & 43.3 & ATP-III & $44.7 \%$ \\
\hline Meyer et al. [13] & USA & Outpatients & $121(60 / 61)$ & 41.1 & ATP-III & $52.1 \%$ \\
\hline Saari et al. [14] & Finland & 1966 Finland & $31(13 / 18)$ & $-{ }^{\mathrm{a}}$ & ATP-III & $19.4 \%$ \\
\hline \multirow[t]{2}{*}{ Koponen et al. [15] } & & birth cohort & & & ATP-IIIA & $29 \%$ \\
\hline & & & & & IDF & $29 \%$ \\
\hline \multirow[t]{2}{*}{ McEvoy et al. [16] } & USA & In-outpatients & $689(180 / 509)$ & 40.4 & ATP-III & $40.9 \%$ \\
\hline & & & & & ATP-IIIA & $42.7 \%$ \\
\hline Hagg et al. [17] & Sweden & In-outpatients & $269(92 / 177)$ & $46^{\mathrm{b}}$ & ATP-III & $34.6 \%$ \\
\hline \multirow[t]{2}{*}{ Meyer et al. [18] } & USA & In-outpatients & $84(6 / 78)$ & 49.0 & ATP-III & $48.8 \%$ \\
\hline & & & & & ATP-IIIA & $56.3 \%$ \\
\hline Correll et al. [19] & USA & Inpatients & $176^{\mathrm{c}}$ & $-{ }^{\mathrm{d}}$ & ATP-III & $37.3 \%$ \\
\hline Lamberti et al. [20] & USA & Outpatients & $93(31 / 62)$ & 34.4 & ATP-IIIA & $53.8 \%$ \\
\hline \multirow[t]{3}{*}{ De Hert et al. [21] } & Belgium & In-outpatients & $430(151 / 279)$ & 36.5 & ATP-III & $28.4 \%$ \\
\hline & & & & & ATP-IIIA & $32.3 \%$ \\
\hline & & & & & IDF & $36 \%$ \\
\hline Teixeira and Rocha [22] & Brazil & Inpatients & $44(10 / 34)$ & 42.2 & ATP-III & $31.8 \%$ \\
\hline $\begin{array}{l}\text { Sanchez-Arana Moreno } \\
\text { et al. [23] }\end{array}$ & Canary Islands & Inpatients & $136(47 / 89)$ & 39.1 & ATP-III & $36 \%$ \\
\hline Tirupati and Chua [24] & Australia & Outpatients & $202(45 / 157)$ & 38.1 & IDF & $69.3 \%$ \\
\hline Srisurapanont et al. [25] & Thailand & Outpatients & $57(33 / 24)$ & 37.5 & IDF & $22.8 \%$ \\
\hline Kurt et al. [26] & Turkey & Inpatients & $296(138 / 158)$ & 55.2 & IDF & $18.9 \%$ \\
\hline Bobes et al. [27] & Spain & Outpatients & $1452(555 / 863)$ & 40.7 & ATP-III & $24.6 \%$ \\
\hline Rejas et al. [28] & & & & & ATP-IIIA & $25.5 \%$ \\
\hline \multirow[t]{2}{*}{ Saddichha et al. [29] } & India & Inpatients & $99(47 / 52)$ & 26.0 & ATP-IIIA & $10.1 \%$ \\
\hline & & & & & IDF & $18.2 \%$ \\
\hline
\end{tabular}


Table 1 continued

\begin{tabular}{|c|c|c|c|c|c|c|}
\hline Study & Country & Sample & $N$ (female/male) & Mean age (years) & Criteria & Prevalence \\
\hline Correll et al. [30] & USA & Inpatients & $111(57 / 54)$ & 44.3 & $\begin{array}{l}\text { ATP-III } \\
\text { ATP-IIIA }\end{array}$ & $\begin{array}{l}45.9 \% \\
54 \%\end{array}$ \\
\hline Cerit et al. [31] & Turkey & Outpatients & $100(41 / 59)$ & 34.7 & $\begin{array}{l}\text { ATP-III } \\
\text { ATP-IIIA } \\
\text { IDF }\end{array}$ & $\begin{array}{l}21 \% \\
34 \% \\
41 \%\end{array}$ \\
\hline Boke et al. [32] & Turkey & Inpatients & $231(57 / 174)$ & 38.5 & IDF & $32 \%$ \\
\hline Oyekcin [33] & Turkey & Outpatients & $34(24 / 10)$ & 33.7 & ATP-III & $35.3 \%$ \\
\hline Kaya et al. [34] & Turkey & Outpatients & $87(36 / 51)$ & 34.4 & $\begin{array}{l}\text { ATP-III } \\
\text { ATP-IIIA } \\
\text { IDF }\end{array}$ & $\begin{array}{l}29.9 \% \\
35.6 \% \\
42.5 \%\end{array}$ \\
\hline Brunero et al. [35] & Australia & Outpatients & $73(28 / 45)$ & 39.3 & IDF & $61.6 \%$ \\
\hline Huang et al. [36] & Taiwan & Outpatients & $650(298 / 352)$ & 45.9 & ATP-III & $34.9 \%$ \\
\hline
\end{tabular}

ATP-III Adult Treatment Protocol of the National Cholesterol Education Program (NCEP) [1], ATP-III A Adapted Adult Treatment Protocol [2, 3], IDF International Diabetes Federation [4]

${ }^{a}$ Mean age 39 years for the whole sample

b Median value

${ }^{c}$ Female/male ratio (169/198) for the whole sample of 367 patients with mixed diagnosis

${ }^{d}$ Mean age 42.9 years for the whole sample of 367 patients with mixed diagnosis 